\title{
Effects of free food deliveries and temporal contiguity on choice under concurrent-chain schedules
}

\author{
Carwyn Scoones, Andrew Hucks, Anthony P. Mclean, and Randolph C. Grace \\ University of Canterbury, Christchurch, New Zealand
}

\begin{abstract}
Eight pigeons responded in a concurrent chain with variable-interval (VI) 10-sec and VI 20-sec terminal links. Free food deliveries were then added to the initial links according to a variable-time (VT) 20 -sec schedule in two conditions that differed in terms of whether a differential reinforcement of other (DRO) contingency was also arranged, which ensured that those deliveries could not occur within 2-sec of a response. Preference for the VI 10-sec terminal link increased when VT food was added, but not when the DRO contingency was operative, showing that free food deliveries affected preference only when those deliveries could be temporally contiguous with choice responding. This finding suggests that Mazur's (2003) report of increased preference with added VT food, replicated here, was due to adventitious reinforcement. Current models for behavioral choice are limited because they are based entirely on temporal relations between stimuli and reinforcers and fail to take into account response-reinforcer contiguity.
\end{abstract}

Research on behavioral choice has studied how nonhumans choose between rewarding outcomes that differ in terms of the immediacy or amount of food reinforcement. This research originated from Herrnstein's (1961) matching law and has led to models for choice that have had substantial impact for understanding human decision making, such as the hyperbolic discounting model for intertemporal choice (see Green \& Myerson, 2004, for review). The question posed by the present study is whether choice between two delayed food rewards is affected by providing additional food deliveries independently of responding.

Our experiment uses a popular paradigm known as concurrent chains, which measures choice between stimuli that signal different rewarding outcomes. In this procedure, subjects (usually pigeons or rats) respond on two alternatives during a choice phase (initial links) to produce access to one of two mutually exclusive outcome schedules (terminal links). The typical result is that subjects respond more to the initial link that leads to the terminal link that provides the more immediate, larger, or more probable reinforcement. Various models for choice in concurrent chains have been proposed, including the contextual choice model (CCM; Grace, 1994), the hyperbolic value added model (HVA; Mazur, 2001), and delay reduction theory (DRT; Fantino, 1969). Although differing in specific details, the equations for these models assume that choice is determined solely by the temporal relations between stimuli and reinforcement. By contrast, there is no explicit role for the relationship between responding and reinforcement, particularly the contingency and temporal contiguity between choice responses and food.

An experiment by Mazur (2003) in which the effect of free food deliveries on choice was investigated is relevant to this issue. In his experiment, pigeons responded on a concurrent-chains schedule in which the initial link was a single variable-interval (VI) 30 -sec schedule that arranged equal access to the terminal links, which were associated with 3-sec and 12-sec delays to food. In some conditions of his experiment, the contingency between responding and reinforcement was degraded by adding a variabletime (VT) 30-sec schedule during the initial links, which provided food independently of responding. Although overall initial-link responding would be expected to decrease during sessions with added VT food because of the degraded contingency (Rachlin \& Baum, 1972), Mazur (2003) showed that the HVA model predicted that choice for the 3-sec alternative should increase. The reason for this prediction was that the average time to reinforcement from the onset of the initial links, which is an important variable in the HVA model, decreased when the VT schedule provided free food. By contrast, according to CCM, there should be no effect of the free food on choice, because its predictions are affected by the time spent in the initial links, which did not change when the VT schedule operated. Mazur (2003) found that results were consistent with HVA: Overall initial-link responding decreased in sessions in which the VT schedule operated but did so relatively more for the 12 -sec outcome, such that the percentage of responses for the 3 -sec outcome increased.

R. C. Grace, randolph.grace@canterbury.ac.nz 
However, an alternative explanation for these results is that the impact of free food deliveries on responding to the preferred key might have been mitigated by adventitious reinforcement. Free food should decrease responding to both initial links because it weakens the contingency between responding and reinforcement (Rachlin \& Baum, 1972). However, if responding to the initial links was differentially affected by the free food, a shift in choice would result. Adventitious reinforcement provides one possible mechanism for responding to a 3 -sec key to have decreased less, resulting in an increased preference. During baseline sessions with no VT food, subjects made more responses to the initial link leading to the 3-sec delay. Thus, when the VT schedule operated, subjects were more likely to be responding to the preferred initial link. As a result, it was more likely for a free food delivery to follow a response to the initial link leading to the 3 -sec delay than the alternative. To the extent that some of these food deliveries were temporally contiguous with responding, responding to the preferred initial link could have been strengthened via adventitious reinforcement, resulting in more extreme preference for the 3-sec alternative.

In our experiment, we planned to test this hypothesis by investigating whether the effects of free food deliveries on choice depended on response contiguity. Specifically, we planned to compare the effects of added VT food, as in Mazur (2003), with the effects of VT food in which there was a differential reinforcement of other (DRO) behavior contingency such that VT food could not be delivered unless at least $2 \mathrm{sec}$ had elapsed since the last initial-link response. Provided that the DRO contingency does not substantially affect the rate of VT food, HVA predicts that preference for the richer alternative should increase to the same extent as when the VT schedule operated without the DRO. However, if adventitious reinforcement was responsible for Mazur's (2003) results, there should be no increase in preference when VT food is provided subject to the DRO contingency.

More broadly, comparing the effects of added VT food both with and without the DRO contingency provides a test of the implicit assumption of models such as HVA, CCM, and DRT that choice is determined solely by the temporal relations between stimuli and reinforcement. If the effect of VT food depends on whether the DRO contingency is operative, models for choice will need to take response-reinforcer contiguity into consideration as an additional factor.

\section{METHOD}

\section{Subjects}

Eight pigeons, numbered $161-164$ and $225-228$, served as subjects. They were maintained at $85 \%$ of their free-feeding weight $\pm 15 \mathrm{~g}$ through appropriate postsession feeding. The subjects were housed individually in a vivarium with a $12: 12 \mathrm{~h}$ light:dark cycle (lights on at $0600 \mathrm{~h}$ ). Water and grit were freely available in the home cages. All the pigeons had prior experimental histories.

\footnotetext{
Apparatus

Eight operant chambers were used; each measured $32 \mathrm{~cm}$ deep $\times$ $34 \mathrm{~cm}$ wide $\times 34 \mathrm{~cm}$ high. Each was enclosed in a sound-attenuating
}

box with which ventilation and white noise were provided by an attached fan; three response keys mounted $21 \mathrm{~cm}$ above the floor were arranged in a row. A force of $0.15 \mathrm{~N}$ was necessary to operate each key. Each chamber had a light located above the center key that provided general illumination and a grain magazine that could be reached through an aperture centered $6 \mathrm{~cm}$ above the floor. During reinforcement, the magazine was operated and illuminated. Experimental events were controlled and the data recorded through a microcomputer and MEDPC interface located in an adjacent room.

\section{Procedure}

Because the subjects were experienced, they were exposed directly to a concurrent-chain procedure. Sessions ended after 48 initial- and terminal-link cycles had been completed or $70 \mathrm{~min}$, whichever occurred first. To signal the initial link at the start of the cycle, the side keys were lighted white. A single VI 10-sec schedule operated during the initial links and arranged access to terminal links. Entry to the terminal links was assigned probabilistically, so that out of every 6 cycles, 3 were to the left key and 3 were to the right key. The VI schedule comprised 12 intervals from an exponential progression (Fleshler \& Hoffman, 1962). A response during the initial link produced entry into the terminal link when (1) it was made to the assigned key, (2) an interval selected from the initial-link schedule had timed out, and (3) a 1-sec changeover delay (COD) had been satisfied - that is, at least $1 \mathrm{sec}$ had elapsed following a changeover to the side where terminal-link entry was arranged. When a terminal link was entered, the color of the appropriate side key would change (left key to red, right key to green), while the other key was darkened. Terminal-link responses were reinforced according to VI 10-sec and VI 20 -sec schedules. The assignment of terminal-link schedules to the left and right keys was counterbalanced. When a response was reinforced, all the lights in the chamber were extinguished, and the grain magazine was raised and illuminated for $2.5 \mathrm{sec}$; then the next cycle began.

There were three conditions in the experiment, which differed only in terms of the contingencies operating in the initial links. The baseline condition consisted of 30 sessions in which the contingencies were those described above. In the VT and VT + DRO conditions, the even-numbered sessions used the same procedure as that for the baseline (i.e., no VT), whereas in the odd-numbered sessions, free food was delivered during the initial links according to a VT 20 -sec schedule. When an interval selected from the VT schedule had elapsed, the grain magazine was raised and illuminated for $2 \mathrm{sec}$ while all other lights were extinguished, and the VI 10-sec schedule was paused. After $2 \mathrm{sec}$ had elapsed, the side keys and houselight were illuminated, and the VI $10-\mathrm{sec}$ schedule resumed. The VT 20 -sec schedule was interrupted after the VI 10-sec schedule timed out and terminal-link entry had been arranged. In the VT + DRO condition, food was made available after the VT 20 -sec schedule had timed out but was delivered only when at least $2 \mathrm{sec}$ had elapsed since the last initial-link response.

All the subjects received the baseline condition first, followed by VT and VT + DRO. For half of the subjects, the VT condition was completed first, followed by VT + DRO, whereas the other half received the opposite order. Between the VT and VT + DRO conditions, all the subjects received 10 additional baseline sessions.

\section{RESULTS}

All the subjects responded more to the initial link leading to the VI 10-sec terminal link by the end of baseline training. Preference for the VI $10 \mathrm{sec}$, measured as the logarithm (base 10) of the initial-link response ratio (VI 10/VI 20 ), was not significantly different in the last five baseline sessions prior to the VT and VT + DRO conditions $[M \mathrm{~s}=$ .58 and .55 , respectively; $S E \mathrm{~s}=.10$ and $.09 ; t(7)=0.76$, n.s.]. This shows that the subjects made approximately 

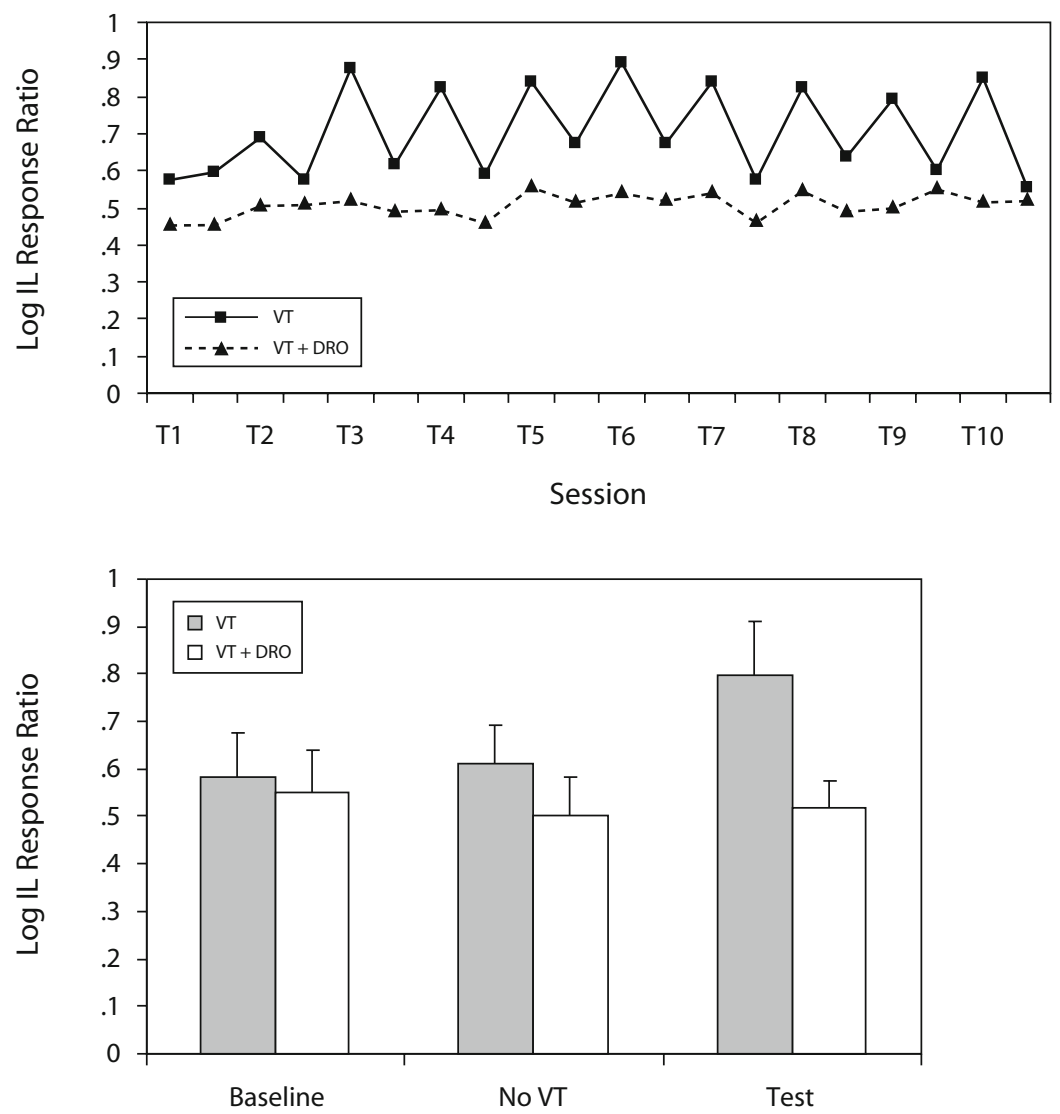

Figure 1. Upper panel: Average log initial-link (IL) response ratios for the preferred key variable-interval ([VI] 10-sec terminal link) for all subjects in the variable-time (VT; squares, solid lines) and VT + differential reinforcement of other (DRO; triangles, dashed lines) conditions. There were 10 pairs of sessions in each condition; VT food was added to the ILs in the first session of each pair (test sessions; T1-T10). Bottom panel: Log response ratios for the last 5 baseline sessions (baseline) and averaged across the 10 sessions in each condition in which VT food was added (test) or was not added (noVT). Bars indicate $+1 S E$.

$80 \%$ of initial-link responses to the VI 10 -sec alternative in baseline sessions.

The primary question was whether the addition of free food during the VT and VT + DRO conditions would affect preference for the VI 10-sec terminal link. The upper panel of Figure 1 shows preference for the VI $10 \mathrm{sec}$ for consecutive test and no-VT sessions in the VT and VT + DRO conditions, averaged across subjects. Overall, preference for the VI $10 \mathrm{sec}$ was stronger during test sessions in the VT condition, but there appeared to be no systematic difference between no-VT and test sessions in the VT + DRO condition. These results were confirmed by a repeated measures ANOVA with condition, session type, and session block (1-10) as factors. There was a significant interaction between condition and session type $[F(1,7)=6.39, p<.05]$. An analysis of simple effects showed that there was a significant difference between test and no-VT sessions for the VT condition, but not for the VT + DRO condition.

The first panel also shows that stable differences between test and no-VT sessions during the VT condition were not obtained until the third session block. Consistent with this, the ANOVA revealed a significant effect of session block $[F(9,63)=3.66, p<.01]$ and an interaction between session block and session type $[F(9,63)=2.22$, $p<.05]$. This result suggests a warm-up effect in the VT condition. Finally, there was a significant effect of condition $[F(1,7)=19.70, p<.01]$, indicating that preference was overall stronger in the VT condition. A linear contrast confirmed that preference was stronger in the VT condition than in the VT + DRO condition during no-VT sessions $[F(1,7)=11.86, p<.05]$.

To provide an overall comparison between the test and no-VT sessions, the bottom panel of Figure 1 shows data averaged across replications of test and no-VT sessions. Data from the baseline sessions prior to testing are also shown. This panel shows that response allocation increased during test, as compared with prior baseline and no-VT sessions, for the VT condition, whereas there was no difference in response allocation between test and both prior baseline and no-VT sessions for the VT + DRO condition. The significant difference for the VT condition, 


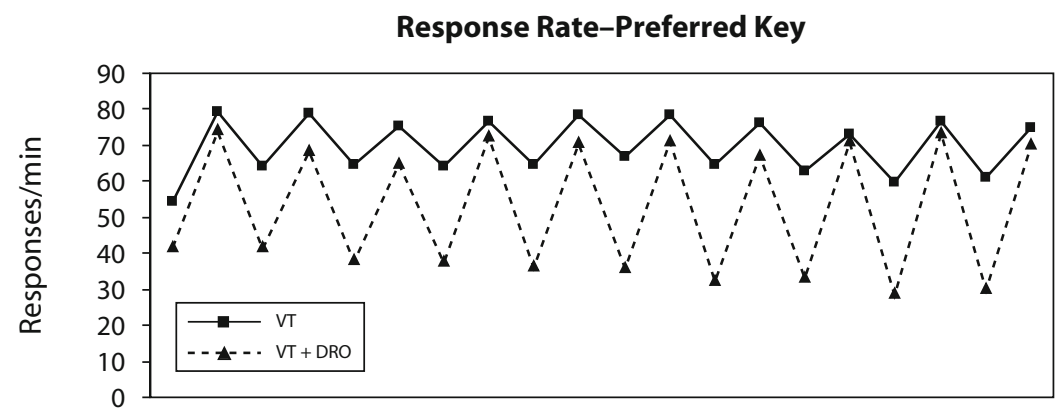

Response Rate-Nonpreferred Key

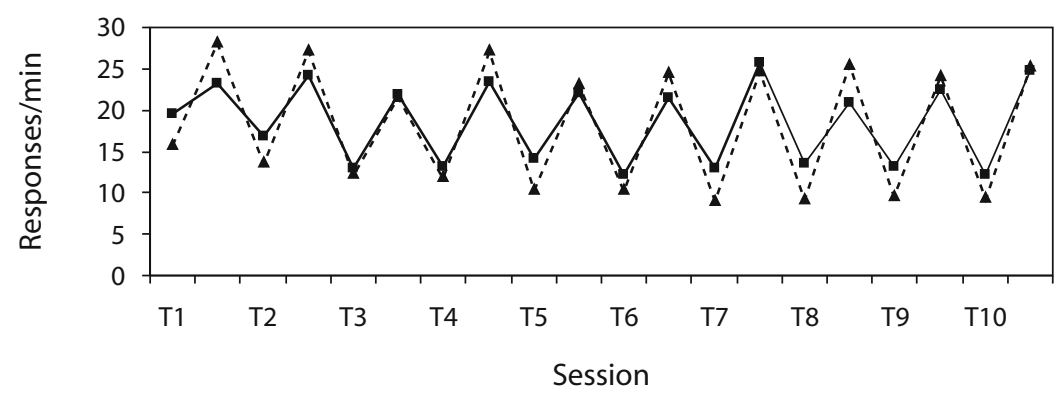

Preferred Key

Nonpreferred Key
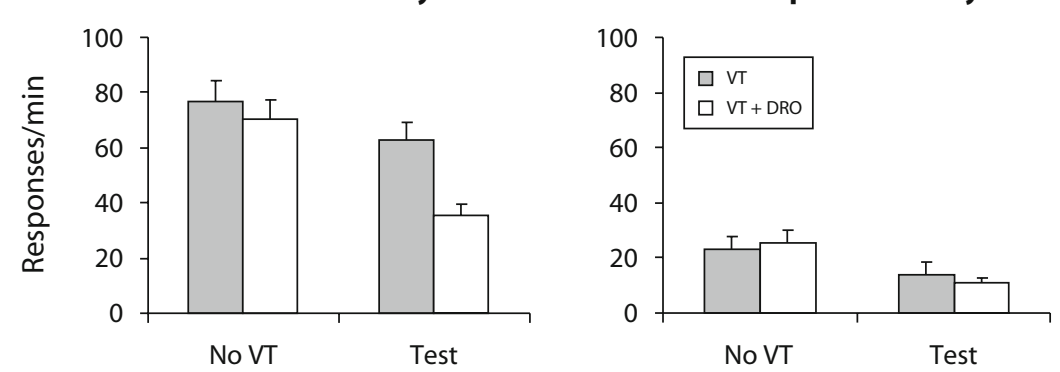

Figure 2. Upper panel: Average initial-link response rates (responses/min) to the preferred key for all sessions in the variable-time (VT; squares, solid lines) and VT + differential reinforcement of other (DRO; triangles, dashed lines) conditions. There were 10 pairs of sessions in each condition; VT food was added to the initial links in the first session of each pair (test sessions; T1-T10). Middle panel: Corresponding data for the nonpreferred key. Lower panel: Response rates for the preferred and nonpreferred keys, averaged across replications of sessions in which VT food was added (test) or not added $(n o V T)$ in each condition. Bars indicate $+1 S E$.

coupled with the lack of difference for the VT + DRO condition, both replicates Mazur's (2003) results and supports the hypothesis that the elevated preference during VT test sessions is attributable to adventitious reinforcement.

To gain a more detailed understanding of how the VT and VT + DRO contingencies affected responding, we analyzed overall response rates to the preferred (i.e., VI 10 -sec) and nonpreferred (i.e., VI 20-sec) initial-link keys. The upper panel of Figure 2 shows response rate to the preferred key for both conditions. A repeated measures ANOVA yielded a significant effect of condition $[F(1,7)=11.16, p<.05]$, indicating that response rates were, overall, greater in the VT condition. A significant effect of session type was also found $[F(1,7)=20.22$, $p<.01]$, indicating that response rates on the preferred key were greater in no-VT than in test sessions. Significant interactions between condition and session type $[F(1,7)=16.91, p<.01]$ and session block and session type $[F(9,63)=2.50, p<.05]$ were also found. The former interaction indicated that the decrease in response rate to the preferred key in test sessions, as compared with noVT, was greater in the VT + DRO condition, whereas the latter showed that the difference in response rates between test and no-VT sessions differed across blocks. Finally, a significant three-way condition $\times$ session block $\times$ session type interaction was obtained $[F(9,63)=2.60, p<.05]$, which showed that whereas the difference in preferred key response rates between test and no-VT sessions did not change systematically across blocks in the VT condition, the difference was larger and increased across blocks in 
the VT + DRO condition. All other effects and interactions did not reach significance.

Response rates to the nonpreferred key are shown in the middle panel of Figure 2. An ANOVA showed a significant effect of session block $[F(9,63)=3.22, p<.01]$, indicating that response rates to the nonpreferred key during the VT and VT + DRO conditions decreased as sessions progressed, which was confirmed by a significant linear contrast $[F(1,7)=12.01, p<.05]$. A significant effect of session type was also found, with $F(1,7)=16.32, p<$ .01 , indicating that response rates were lower in the test sessions. None of the interactions were significant, suggesting that responding decreased to a similar extent in the VT and VT + DRO test sessions.

These results are summarized in the bottom panels of Figure 2, which show response rates to the preferred and nonpreferred keys averaged across replications of test and no-VT sessions. These show that response rates to the preferred key (left panel) decreased more during test sessions in the VT + DRO condition than in VT test sessions. By contrast, response rates to the nonpreferred key (right panel) decreased approximately to the same extent during test sessions in both conditions. These results support the adventitious reinforcement hypothesis, because responding on the preferred key was more likely to be maintained by free food delivery.

Finally, we checked whether the obtained rates of VT reinforcement were different in the VT and VT + DRO conditions, an analysis of obtained rate of reinforcement was undertaken. Table 1 shows the overall VT reinforcement rates for each subject, as well as the average reinforcement rates and standard errors. On average, 102 and 98 free food deliveries per minute occurred during the initial links for test sessions in the VT and VT + DRO conditions, respectively, which were not significantly different $[t(7)=0.80$, n.s. $]$. Thus, differences in responding between the conditions were not confounded by differences in the obtained rates of free food in the initial links.

\section{DISCUSSION}

This experiment showed that free food deliveries added to the initial links of a concurrent-chain procedure increased preference for the key leading to the shorter delay

\section{Table 1}

Obtained Rates of Reinforcement (per Minute) During Variable Time (VT) and VT + Differential Reinforcement of Other (DRO) Conditions (With the Average Rates and Standard Errors for Each Condition)

\begin{tabular}{crr}
\hline Subject & \multicolumn{1}{c}{ VT } & VT + DRO \\
\hline Bird 161 & 133.44 & 105.85 \\
Bird 162 & 114.67 & 99.09 \\
Bird 163 & 83.27 & 94.70 \\
Bird 164 & 99.07 & 97.15 \\
Bird 225 & 110.08 & 100.32 \\
Bird 226 & 87.24 & 98.42 \\
Bird 227 & 105.16 & 103.56 \\
Bird 228 & 85.57 & 89.07 \\
Average & 102.31 & 98.52 \\
$S E$ & 6.08 & 1.83 \\
\hline
\end{tabular}

to reinforcement only when those deliveries could occur in close temporal contiguity with choice responses. When the free food deliveries were provided at the same rate but could not occur for at least $2 \mathrm{sec}$ after a choice response, there was no systematic change in preference. The free food reduced response rates overall, as was expected, because it degraded the contingency between responding and reinforcement (Rachlin \& Baum, 1972). Whereas response rates to the nonpreferred key decreased to the same extent when free food deliveries occurred in both the VT and VT + DRO conditions, response rates to the preferred key were reduced less in the VT than in the VT + DRO condition. Consequently, choice for the preferred key increased in the VT condition-replicating Mazur (2003) - but not in the VT + DRO condition.

The most likely explanation for why the response rate to the preferred key was reduced less in the VT condition was that it occurred because of adventitious reinforcement. Because the subjects were already responding more to the preferred key, free food deliveries were more likely to have closely followed a response to that key than to the alternative. Accidental temporal contiguity could have strengthened responding to the preferred key via adventitious reinforcement, thus mitigating, to some extent, the reduction in responding produced by the VT food. The results from the VT + DRO condition showed that when such accidental contiguity was prevented, responding decreased substantially on both keys and proportionally by about the same extent, such that there was no systematic change in preference although free food deliveries occurred at the same rate.

Note that this process of adventitious reinforcement suggests a positive feedback loop; that is, stronger preference makes it relatively more likely that the free food deliveries will follow a response to the preferred than to the nonpreferred initial link, increasing the probability that preferred key responding will be strengthened by adventitious reinforcement, leading to a further increase in preference. Thus, the impact of VT food on preference should increase over time, and the data in Figure 1 were consistent with this expectation.

Our finding that free food deliveries added to the initial links of concurrent chains increase preference only when those deliveries can be temporally contiguous with responding has implications for models of choice behavior. This suggests that the increased preference reported by Mazur (2003) was also due to adventitious reinforcement. Our results pose a challenge to accounts of choice, such as Mazur's (2001) HVA, which assume that the time to food from the onset of the initial links is a determiner of preference. Because the average time to food from initial-link onset decreased to the same extent in the VT and VT + DRO conditions, HVA predicts that preference should have increased similarly in both conditions. Thus, the failure to find an increase in preference during the VT + DRO test sessions is contrary to HVA - specifically, its assumption that the primary determiner of choice is the value of the initial- and terminal-link stimuli, with value calculated as a function of delays to reinforcement. Similarly, our results are contrary to DRT (Fantino, 1969). Ac- 
cording to DRT, preference is determined by the relative conditioned reinforcing value of the terminal-link stimuli, which is a function of the average time to food from the initial and terminal links. Because the former decreased similarly in both conditions, DRT makes the same predictions as HVA and, thus, also fails to predict the lack of increased preference in the VT + DRO condition.

In contrast, models that assume that the average duration of the initial links is a determiner of choice, such as CCM (Grace, 1994), predict that the free food deliveries used here and by Mazur (2003) should not affect choice and, thus, are consistent with the results from the VT + DRO condition. More broadly, however, our finding that adventitious reinforcement can affect choice suggests that all current models for choice are limited. The equations for HVA, DRT, and CCM assume that choice is determined strictly by the temporal relations between stimuli and reinforcement, which determine stimulus value. The present results show that the contiguity between responses and reinforcement is also an important factor that affects choice (cf. Bell, 1999; Grace, Schwendiman, \& Nevin, 1998). The implication is that current models for choice are incomplete, since they fail to take into account the temporal contiguity of responding and reinforcers. Future research should address this limitation by systematically exploring the effects of additional food deliveries during the initial links.

Finally, our results suggest that the relative value of the terminal links is unaffected by providing free food during the initial links. From a behavioral economic perspective, if the free-food deliveries are interpreted as an increase in income, this finding stands in contrast to studies that have shown that nonhumans' preference between alternatives that differ in terms of delay, amount, or probability of reward depends on income level. For example, Hastjarjo and Silberberg (1992) found that rats' preference for a delayed alternative that provided three food pellets over one that provided a single food pellet immediately increased when the overall number of trials in a session was reduced. Similar results were reported by Hastjarjo, Silberberg, and Hursh (1990) for rats choosing between certain and risky rewards. These studies suggest that organisms are sensitive to the overall amount of income gained and that how delay, amount, and probability of reward determine value changes depending on income level. By contrast, our results indicate that how relative value is determined by delay was unaffected by free food. Whether this discrepancy indicates that the addition of VT food and changes in trials per session are not equivalent ways of manipulating "income," or may be explained in terms of species differences (pigeons vs. rats) or other procedural parameters, is a question for future research.

\section{AUTHOR NOTE}

Address correspondence to R. C. Grace, Department of Psychology, University of Canterbury, Private Bag 4800, Christchurch, New Zealand (e-mail: randolph.grace@canterbury.ac.nz).

\section{REFERENCES}

Bell, M. C. (1999). Pavlovian contingencies and resistance to change in a multiple schedule. Journal of the Experimental Analysis of Behavior, 72, 81-96.

FAntino, E. (1969). Choice and rate of reinforcement. Journal of the Experimental Analysis of Behavior, 12, 723-730.

Fleshler, M., \& Hoffman, H. S. (1962). A progression for generating variable-interval schedules. Journal of the Experimental Analysis of Behavior, 5, 529-530.

Grace, R. C. (1994). A contextual model of concurrent-chains choice. Journal of the Experimental Analysis of Behavior, 61, 113-129.

Grace, R. C., Schwendiman, J. I., \& Nevin, J. A. (1998). Effects of delayed reinforcement on preference and resistance to change. Journal of the Experimental Analysis of Behavior, 69, 247-261.

Green, L., \& Myerson, J. (2004). A discounting framework for choice with delayed and probabilistic rewards, Psychological Bulletin, 130, 769-792.

Hastjarjo, T., \& Silberberg, A. (1992). Effects of reinforcer delays on choice as a function of income level. Journal of the Experimental Analysis of Behavior, 57, 119-125.

Hastjarjo, T., Silberberg, A., \& Hursh, S. R. (1990). Risky choice as a function of amount and variance in food supply. Journal of the Experimental Analysis of Behavior, 53, 155-161.

HerRnsteIn, R. J. (1961). Relative and absolute strength of response as a function of frequency of reinforcement. Journal of the Experimental Analysis of Behavior, 4, 267-272.

Mazur, J. E. (2001). Hyperbolic value addition and general models of animal choice. Psychological Review, 108, 96-112.

Mazur, J. E. (2003). Effects of free-food deliveries and delays on choice under concurrent-chains schedules. Behavioural Processes, 64, 251260.

RACHLIN, H., \& BAUM, W. M. (1972). Effects of alternative reinforcement: Does the source matter? Journal of the Experimental Analysis of Behavior, 18, 231-241.

(Manuscript received January 22, 2009; revision accepted for publication March 25, 2009.) 